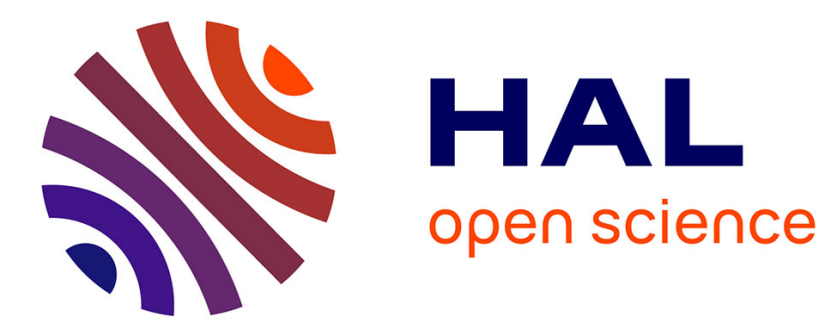

\title{
Defining a proxy for the interpretation of seismic anisotropy in non-Newtonian mantle flows
}

\author{
N. Hedjazian, E. Kaminski
}

\section{To cite this version:}

N. Hedjazian, E. Kaminski. Defining a proxy for the interpretation of seismic anisotropy in non-Newtonian mantle flows. Geophysical Research Letters, 2014, 41, pp.7065-7072. 10.1002/2014GL061372 . insu-03581072

\section{HAL Id: insu-03581072 \\ https://hal-insu.archives-ouvertes.fr/insu-03581072}

Submitted on 19 Feb 2022

HAL is a multi-disciplinary open access archive for the deposit and dissemination of scientific research documents, whether they are published or not. The documents may come from teaching and research institutions in France or abroad, or from public or private research centers.
L'archive ouverte pluridisciplinaire HAL, est destinée au dépôt et à la diffusion de documents scientifiques de niveau recherche, publiés ou non, émanant des établissements d'enseignement et de recherche français ou étrangers, des laboratoires publics ou privés. 


\section{Geophysical Research Letters}

\section{RESEARCH LETTER \\ 10.1002/2014GL061372}

Key Points:

- We consider non-Newtonian flows consistent with dislocation creep

- Seismic anisotropy rises from plastic deformation and dynamic recrystallization

- We define a proxy based on finite strain to interpret seismic anisotropy

Correspondence to:

N. Hedjazian,

hedjazia@ipgp.fr

\section{Citation:}

Hedjazian, N., and E. Kaminski (2014), Defining a proxy for the interpretation of seismic anisotropy in non-Newtonian mantle flows, Geophys. Res. Lett., 41, 7065-7072, doi:10.1002/2014GL061372.

Received 29 JUL 2014 Accepted 29 SEP 2014 Accepted article online 2 OCT 2014 Published online 23 OCT 2014

\section{Defining a proxy for the interpretation of seismic anisotropy in non-Newtonian mantle flows}

\author{
N. Hedjazian ${ }^{1}$ and E. Kaminski ${ }^{1}$ \\ ${ }^{1}$ Institut de Physique du Globe de Paris, Sorbonne Paris Cité, Université Paris Diderot, CNRS, Paris, France
}

Abstract Seismic anisotropy can provide unique insights on convection in the upper mantle. Here we study the link between seismic anisotropy and mantle flow using a non-Newtonian rheology consistent with deformation by dislocation creep. Using analytical first-order flow models underneath a ridge and in subduction zones, we find that finite strain ellipsoid (FSE) is a robust proxy of seismic anisotropy, both in terms of orientation and strength, for natural strains smaller than $\approx 1$. At larger strains, anisotropy aligns with the "infinite strain axis" (ISA), defined as the orientation of the long axis of the FSE in the limit of infinite strain, and its percentage reaches a plateau. Anisotropy aligns with the flow direction only when the product of the inverse strain rate with the timescale of ISA rotation within the flow is smaller than 0.1 .

\section{Introduction}

Seismic anisotropy in the Earth's upper mantle is a key tool to infer the geometry and organization of convective flow [e.g., Tanimoto and Anderson, 1984; Montagner, 1994; Savage, 1999]. Olivine is the major and most anisotropic constituent of the upper mantle, and its compressional wave velocity can vary up to $20 \%$ with direction [Kumazawa and Anderson, 1969; Nicolas and Christensen, 1987]. The hypothesis underlying the interpretation of seismic anisotropy in terms of mantle flow is that it results from lattice preferred orientation (LPO) of olivine crystals when it is consistent at the (regional) scale of the convective flow. Whereas olivine crystals are orthorhombic, the seismic anisotropy produced by their LPO is most often described in terms of hexagonal symmetry (also called transverse isotropy) [e.g., Montagner and Nataf, 1988]. Seismic observables, such as SKS splitting measurements or surface wave dispersion, can be used to infer the direction of a symmetry axis and a percentage of anisotropy corresponding to the difference between fast and slow propagation velocities.

The interpretation of anisotropy can be controversial, depending on various underlying hypotheses on the relationships between the direction of the LPO and either the flow direction or a direction characteristic of the deformation in the area of interest. For example, recent studies have used the principal axis of the strain rate tensor [Gaboret et al., 2003], or the absolute plate motion direction [Kreemer, 2009]. The relationship between anisotropy and deformation in the flow can be made explicit by studying the LPO development through the deformation of olivine polycrystals. A complete characterization of LPO, hence of seismic anisotropy, can be obtained using mechanical models of polycrystal deformation, such as the ones based on the viscoplastic self-consistent theory (VPSC), coupled with a computed mantle flow [e.g., Tommasi, 1998; Blackman and Kendall, 2002; Di Leo et al., 2014]. However, a complete forward modeling of seismic anisotropy is numerically demanding, especially for 3-D and time-varying flows, and simple proxies remain useful for the interpretation of seismic anisotropy [e.g., Lev and Hager, 2008].

At low strain and for uniform deformation, experiments [Nicolas et al., 1973; Zhang and Karato, 1995] and theoretical models [Ribe, 1992] showed that LPO (hence seismic anisotropy) can be simply described in terms of finite strain, the olivine fast axis being aligned with the long axis of the finite strain ellipsoid (FSE), and the percentage of anisotropy being a monotonic function of the ratio of the short and long axes of the FSE. Based on these results, the FSE has been used as a proxy to interpret seismic anisotropy in terms of large-scale mantle flow [e.g., Hall et al., 2000; Becker et al., 2006; Li et al., 2014].

At large stain, texture evolution is not only due to internal crystal deformation but also and mainly controlled by dynamic recrystallization (subgrain rotation, grain nucleation, grain boundary migration, and grain boundary sliding) [Poirier, 1985]. Simple shear deformation experiments showed that dynamic recrystallization makes the LPO rotate from the long axis of the FSE to the direction of shear [Zhang and Karato, 1995; Bystricky et al., 2000]. Kaminski and Ribe [2001, 2002] developed a theoretical model of dynamic 
recrystallization in olivine polycrystals to reproduce the experimental observations, and they extrapolate their results to viscous mantle flows. They obtained that dynamic recrystallization will tend to align the fast axis of the olivine crystals with the infinite strain axis (ISA), defined as to the orientation of the long axis of the FSE in the limit of infinite strain. Kaminski and Ribe [2002] further introduced a dimensionless parameter that could be used to identify the area in which anisotropy can be simply related to the flow direction. This parameter, called the grain orientation lag $(\mathrm{GOL})$, is defined as the ratio between the timescale of LPO evolution (which is the inverse of the local strain rate) and the rate of rotation of the ISA along the flow lines. For values of $\mathrm{GOL} \ll 1$, the direction of fast propagation in the anisotropic medium is the same as the flow direction. This criterion has been used, for example, to study the relationship between flow models and seismic anisotropy in the upper mantle [e.g., Conrad et al., 2007; Conrad and Behn, 2010].

The GOL parameter criterion was established by Kaminski and Ribe [2002] using Newtonian flows. This hypothesis is not fully consistent, as LPO initially arises from plastic deformation of the olivine crystals, which, by definition, corresponds to a non-Newtonian (power law) rheology. The aim of the present paper is to study the relationship between seismic anisotropy, FSE, and ISA in the case of non-Newtonian flows and to discuss their potential use as proxies of seismic anisotropy. To that aim, we will follow a systematic approach relying on analytical solutions of the Stokes equations for a power law rheology and in two main geodynamic environments, underneath mid-oceanic ridges and in subduction zones.

\section{Methods: LPO Evolution in Viscous Non-Newtonian Flows}

\subsection{Non-Newtonian Corner Flows as Analogue of Mantle Flow in a Subduction Zone and Beneath a Ridge}

Seismic anisotropy is a function of the deformation history of the olivine aggregates integrated along the flow lines. To assess the relationship between the characteristics of anisotropy and the characteristics of the flow, we choose an analytical approach and used simple corner flows that (i) encompass some of the main features of plate driven mantle flows, in subduction zones and below ridges, and (ii) allow a fast calculation of the LPO evolution. We consider two families of corner flows that are solutions of the Stokes equations for a viscous fluid and at low Reynolds number [Batchelor, 1967],

$$
\begin{gathered}
\nabla \cdot \mathbf{u}=0, \\
\nabla \cdot 2 \eta \mathrm{e}=\nabla \mathbf{p},
\end{gathered}
$$

where $\mathbf{u}, \mathrm{e}, \mathbf{p}$, and $\eta$ are the flow velocity, the strain rate tensor, the pressure, and the fluid viscosity, respectively. The Stokes equation is solved for a power law rheology consistent with deformation by dislocation creep, such that [Ribe, 1989]

$$
\eta=\eta_{0}\left(\frac{I_{2}}{\mathrm{e}_{0}}\right)^{\frac{1}{n}-1},
$$

where $I_{2}$ is the second invariant of the stain rate tensor, $\eta_{0}$ is the viscosity at the reference strain rate $e_{0}$, and $n$ is the stress exponent characterizing the rheology. A Newtonian rheology corresponds to $n=1$, whereas the rheology corresponding to dislocation creep of olivine yields $n \approx 3.5-3.6$ [Bai et al., 1991; Jin et al., 1994]. In the following, we will take $n=3$ as an approximation for dislocation creep of olivine in order to get analytical solutions. Note that this model is an analogue of viscous flow in the mantle and that further refinements will be necessary to study the "frozen-in" LPO, e.g., through temperature-dependent viscosity. Because these flows are steady, they may not account for all the deformation patterns in nonsteady state subduction zones.

The analytical solutions for the (Newtonian and non-Newtonian) flows in polar coordinates $(r, \theta)$ are expressed using a dimensionless stream function $\Psi=r f(\theta)$, such that $\left(u_{r}, u_{\theta}\right)=U_{0}\left(f^{\prime},-f\right)$, with $U_{0}$ the velocity of the plate driving the flow [Batchelor, 1967]. The form of the function $f(\theta)$ depends on the mechanical boundary conditions and on the exponent of the power law rheology and is written as follows [Tovish et al., 1978]:

$$
\begin{gathered}
f(\theta)=A \sin \theta+B \cos \theta+C \sin \theta+D \cos \theta, \quad \text { for } n=1, \\
f(\theta)=A \sin \theta+B \cos \theta+C h(\theta, D), \quad \text { for } n=3,
\end{gathered}
$$


Table 1. Analytical Solutions ${ }^{\mathrm{a}}$ for the Stream Function $\Psi=r f(\theta)$

\begin{tabular}{|c|c|c|c|c|}
\hline & \multicolumn{2}{|c|}{ Mid-Ocean Ridge } & \multicolumn{2}{|c|}{ Subduction Zone } \\
\hline & $n=1$ & $n=3$ & $n=1$ & $n=3$ \\
\hline$A$ & 0 & $-C h(\pi / 2)$ & $\left(\theta_{0} \sin \theta_{0}\right) /\left(\theta_{0}^{2}-\sin ^{2} \theta\right)$ & $-C h^{\prime}(0)$ \\
\hline$B$ & 0 & 0 & 0 & $-C h(0)$ \\
\hline$C$ & 0 & $\left(h^{\prime}(\pi / 2)\right)^{-1}$ & $\left(\theta_{0} \cos \theta_{0}-\sin \theta_{0}\right) /\left(\theta_{0}^{2}-\sin ^{2} \theta\right)$ & $\left(h(0) \sin \theta_{0}-h^{\prime}(0) \cos \theta_{0}+h^{\prime}\left(\theta_{0}\right)\right)^{-1}$ \\
\hline$D$ & $-2 / \pi$ & $3 \pi /(2 \sqrt{5})$ & $-A$ & $h\left(\theta_{0}\right)=\sin \theta_{0} h^{\prime}(0)-\cos \theta_{0} h(0)$ \\
\hline
\end{tabular}

aThe function $h$ is the one defined in equation (6). In the case of the non-Newtonian flow analogue to a subduction zone, $D$ is solution of a transcendental equation.

with

$$
h(\theta)=27 \cos \frac{\sqrt{5}}{3}(\theta+D)-\cos \sqrt{5}(\theta+D)
$$

where $A, B, C$, and $D$ are determined from the boundary conditions (Table 1 ).

Figure 1 shows a few examples of flow lines for the two corner flows and for Newtonian and non-Newtonian rheologies. The non-Newtonian rheology produces a velocity field similar to the one produced with a Newtonian rheology in the two geometries. However, streamlines tend to tighten up with increasing values of $n$, leading to stronger velocity gradients close to the plate driving the flow. It is thus likely that the differences between Newtonian and non-Newtonian cases in terms of seismic anisotropy, which is controlled by the deformation of the aggregates integrated along the flow lines, are going to be more significant than the difference between the flow lines themselves.

\subsection{Crystal Deformation and Dynamic Recrystallization}

LPO evolution in the flows (hence anisotropy) results from the progressive deformation along the flow lines of the crystal aggregates subject to the local velocity gradient tensor $L=\nabla \mathbf{u}$. In the analytical cases considered here, $\mathrm{L}$ is given by

$$
\mathrm{L}_{r \theta}=\frac{f+f^{\prime \prime}}{r}\left(\begin{array}{ll}
0 & 1 \\
0 & 0
\end{array}\right)
$$
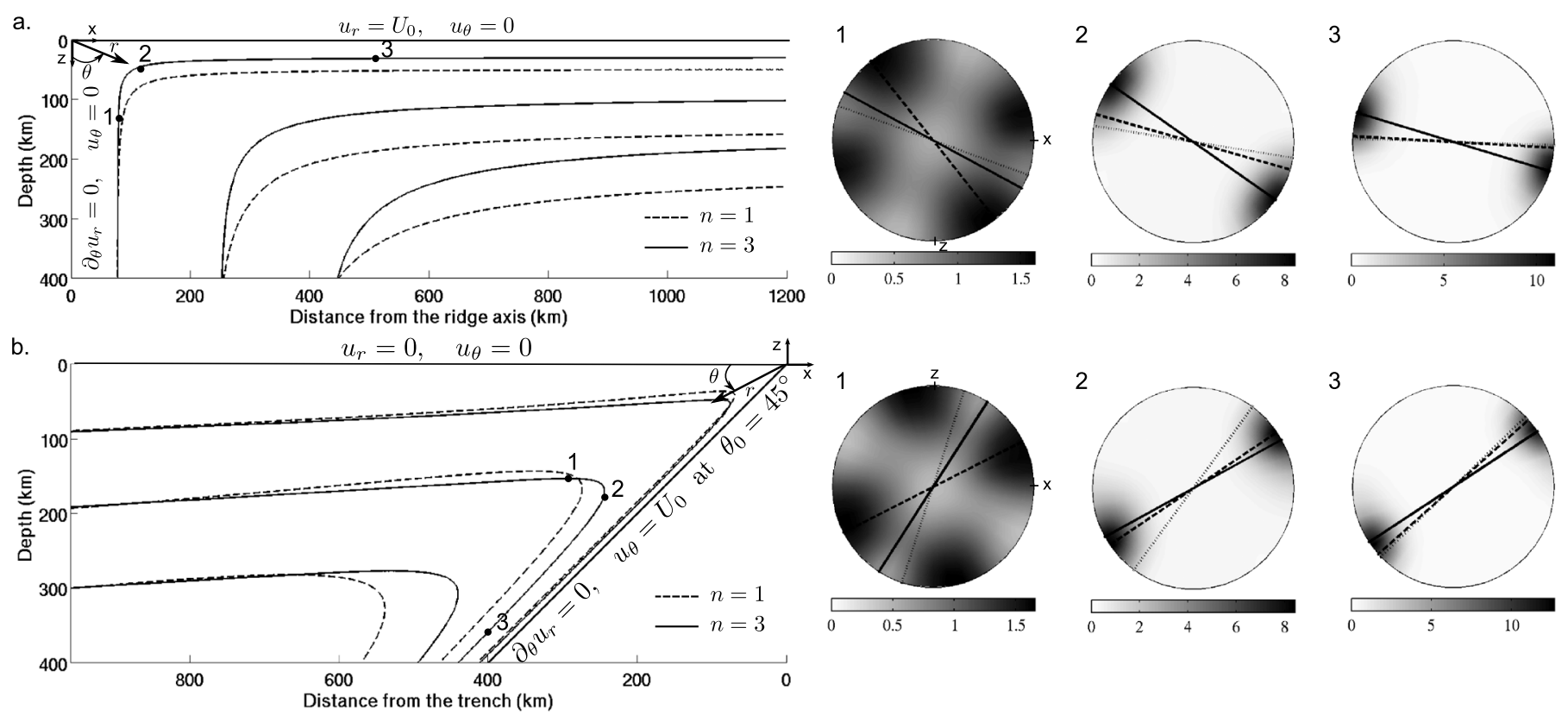

Figure 1. Three examples of streamlines in 2-D corner flows taken as first-order approximation of (a) a mid-ocean ridge, (b) a subduction zone, and for two different rheologies (solid lines: non-Newtonian rheology, $n=3$; dashed lines: Newtonian rheology, $n=1$ ). Pole figures correspond to lower hemisphere projections of [100] olivine lattice, in multiples of uniform distribution, at three locations along two streamlines in the non-Newtonian flows. Solid lines show the hexagonal symmetry axis, dashed lines the ISA, and dotted lines the FSE long axis. 

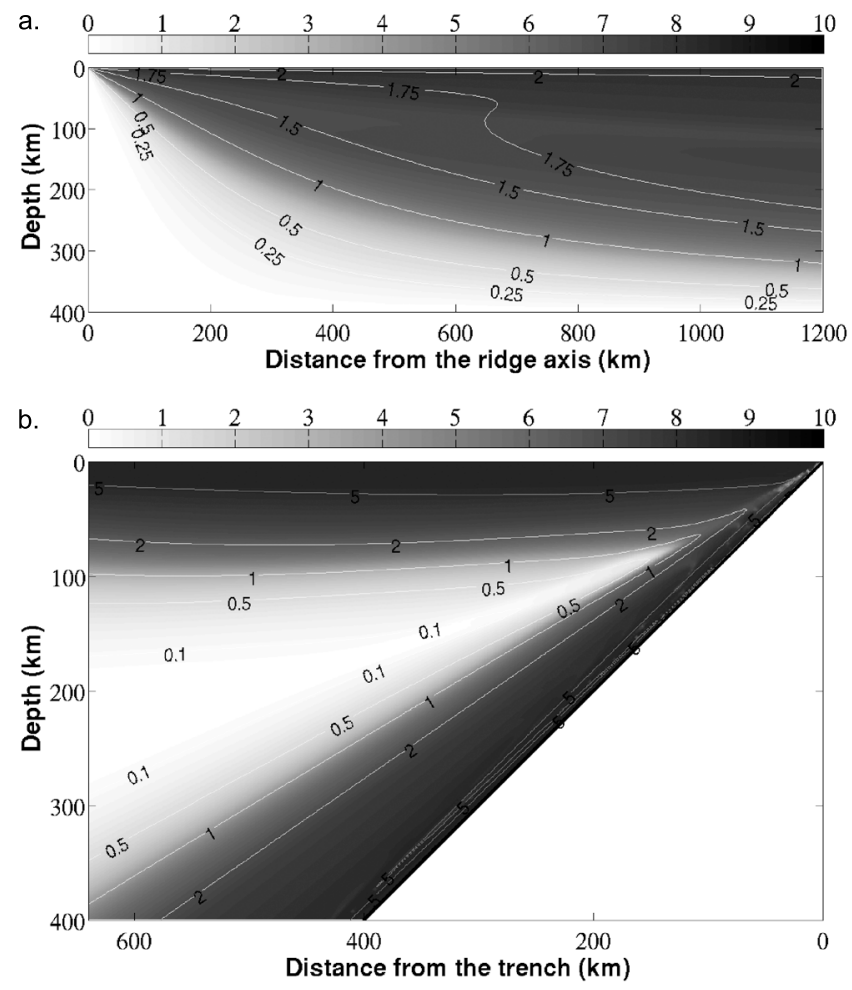

Figure 2. Percentage of anisotropy in the hexagonal medium that best fits the local elastic tensor (gray scale map) compared to the natural strain (level lines) for a power law rheology, with a grain boundary mobility $M^{*}=125$, and for (a) a mid-ocean ridge and (b) a subduction zone. which shows that the local deformation at an arbitrary point $(r, \theta)$ corresponds to a simple shear on a plane normal to the plane $(r, \theta)$ and with a shear direction along $\theta$. The deformation produced by the integration of the local deformation along the flow lines is fully described by the finite strain ellipsoid (FSE) [McKenzie, 1979]. Within the FSE framework, a random distribution of orientations in a polycrystal aggregate corresponds to a sphere. The sphere later becomes an ellipsoid as a function of the imposed deformation. The relevant parameters for seismic anisotropy are the orientation of the long axis of the FSE, and the natural strain, $E_{0}=(1 / 2) \ln (a / c)$, with $a$ and $c$ the lengths of the major and minor axes of the ellipsoid, respectively.

To further model LPO evolution by plastic deformation and dynamic recrystallization in the flows, we used D-Rex, the model developed by Kaminski et al. [2004]. In this model, the LPO evolves by four mechanisms: internal plastic deformation of the crystals, grain nucleation, grain

boundary migration, and grain boundary sliding. The model parameters are the activity of the slip systems for olivine and enstatite, a dimensionless nucleation rate, a dimensionless grain boundary mobility, and a dimensionless activation volume for grain boundary sliding. All the parameters are constrained by comparison with laboratory experiments [Nicolas et al., 1973; Zhang and Karato, 1995; Bystricky et al., 2000]. In the following, we consider an aggregate of $N=17^{3} \approx 5000$ crystals with $70 \%$ olivine and $30 \%$ enstatite. We assume that olivine crystals deform by dislocation creep on three independent slip systems, (010)[100], (001)[100], and (010)[001]. The respective normalized reference resolved shear stresses are $\tau_{0}^{(010)[100]}=1$, $\tau_{0}^{(001)[100]} / \tau_{0}^{(010)[100]}=2$, and $\tau_{0}^{(010)[001]} / \tau_{0}^{(010)[100]}=3$, which will result in the most common "type $\mathrm{A}^{\text {" olivine }}$ LPO [e.g., Karato et al., 2008]. Parameters for dynamic recrystallization are the same as in Kaminski et al. [2004]. Once the texture evolution has been calculated, the elastic tensor is obtained by a Voigt average and decomposed in the different symmetry classes as in Browaeys and Chevrot [2004]. We then quantify seismic anisotropy using the hexagonal medium that best fits the full elastic tensor. The hexagonal symmetry axis corresponds to the direction of fast propagation that we will refer to as "the fast axis" in the following, and the module of the hexagonal part of the elastic tensor gives the percentage of anisotropy.

\section{Results: Defining a Proxy of Seismic Anisotropy in Non-Newtonian Mantle Flows 3.1. The Relationship Between Finite Strain and Seismic Anisotropy}

As a first step, we evaluate the relevance of FSE as a proxy for seismic anisotropy, since they have been shown to be closely related in the case of uniform deformations at moderate strain [Ribe, 1992]. We first compare the percentage of anisotropy with the natural strain in the two flows (ridge and subduction zone). The results, shown in Figures 2 and 3, demonstrate that natural strain provides a satisfying estimate of the percentage of anisotropy, up to a finite strain $E_{0} \approx 1$. For a given value of the natural strain smaller than 1 , the percentage of anisotropy increases for larger grain boundary mobility. For larger strains, anisotropy reaches a plateau value of $\approx 8 \%$. The maximum strain at which the plateau value is reached decreases with increasing grain boundary mobility. This plateau corresponds to a "steady state" LPO in which the 

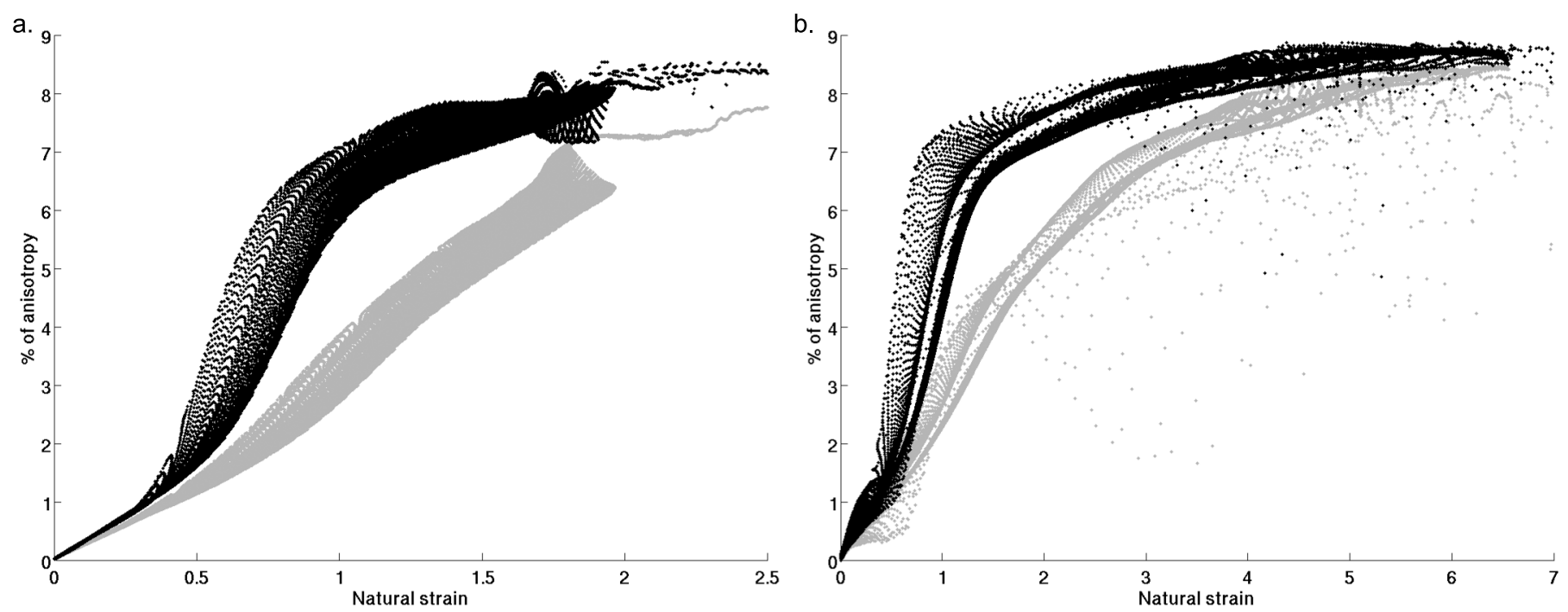

Figure 3. Percentage of anisotropy in the hexagonal medium that best fits the local elastic tensor as function of the natural strain for two extreme values of grain boundary mobility (gray: $M^{*}=50$; black: $M^{*}=250$ ) and for (a) a ridge and (b) a subduction zone. Each dot on the graph corresponds to a calculation at a grid point of the model.

orientations of the crystals are distributed around a single average orientation, with a small random deviation from the average orientation due to grain boundary sliding, and with the fast axis of enstatite perpendicular to the fast axis of olivine [Kaminski et al., 2004].

We then compare the orientation of the FSE long axis with that of the fast axis of the hexagonal medium that best fits the elastic tensor. Figure 4 shows the mismatch between the two orientations. At small strains
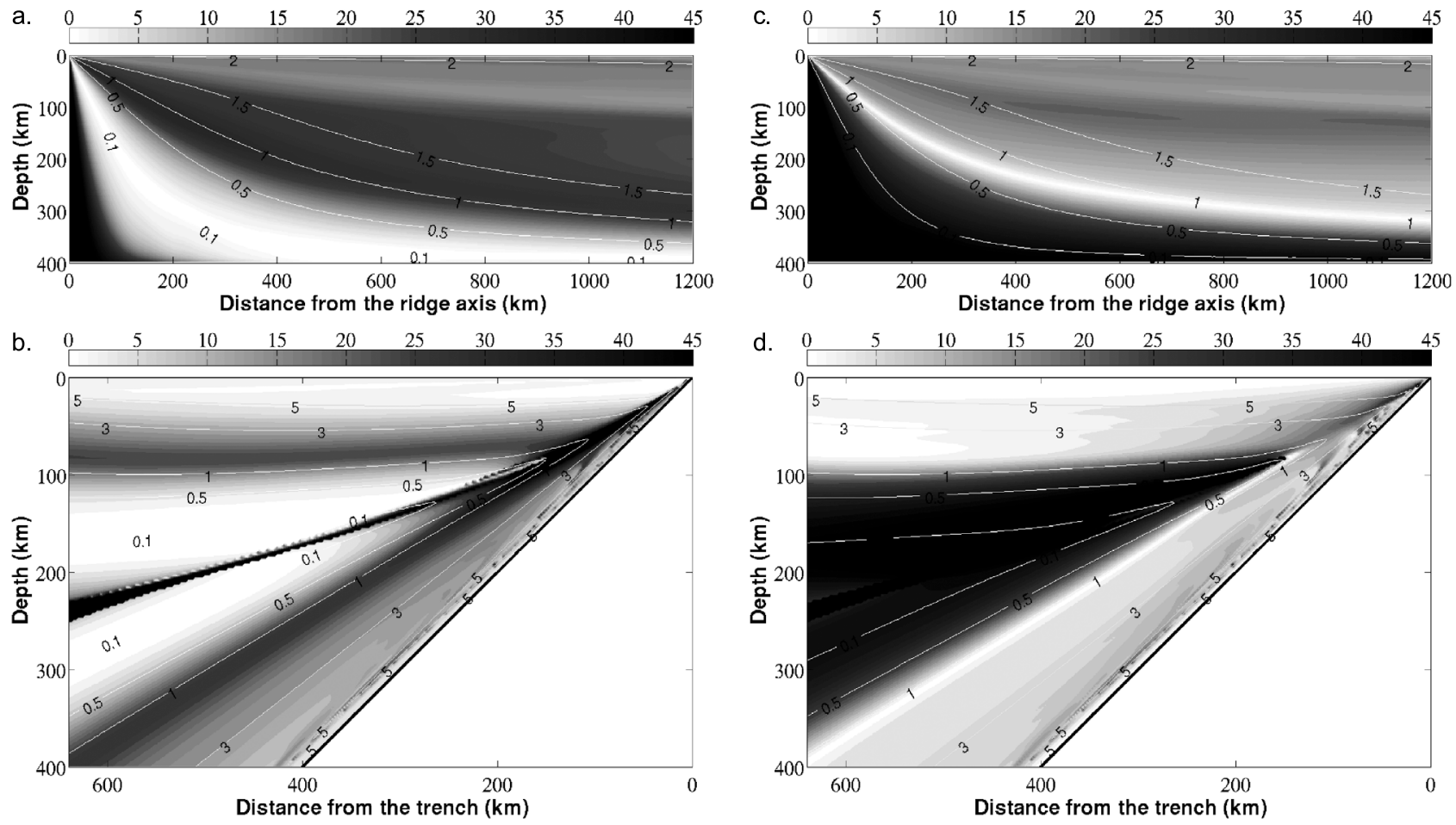

Figure 4. Evolution of the angle between the orientation of FSE long axis and the hexagonal symmetry axis (gray scale, saturated at $45^{\circ}$ ) as a function of the natural strain (given by the contour lines): (a) Mid-ocean ridge and (b) subduction zone. Inconsistent values arise when $E_{0}<0.1$, which is a too small deformation for the hexagonal symmetry to be a good approximation of the local elastic tensor. Angle between the ISA and the hexagonal symmetry axis (gray scale, saturated at $45^{\circ}$ ), as a function of the natural strain (given as contour lines): (c) Mid-ocean ridge and (d) subduction zone. 
$\left(E_{0}<0.5\right)$, the FSE long axis has the same orientation as the hexagonal symmetry axis. For strains $E_{0}>0.5$, the accumulated deformation is large enough for dynamic recrystallization to control the evolution of LPO and the FSE evolution starts to lag behind the LPO. The difference between the two orientations can be quite small in the case of a flow in which the direction of shear does not vary much along the flow lines but can be large in the case of rapid rotation of the streamlines. This is consistent with the results of Faccenda and Capitanio [2013], who have calculated an average mismatch angle of $22^{\circ}$ using 3-D dynamic models of subduction. Where $0.5<E_{0}<1$, the difference remains smaller than $15^{\circ}$ and the FSE remains an acceptable proxy. For $E_{0}>1$, it may be more relevant to use the ISA as a proxy for the orientation of the fast axis [Kaminski and Ribe, 2002], as discussed below.

\subsection{Direction of Anisotropy and Orientation of the Infinite Strain Axis}

In crystal aggregates deformed under uniform simple shear in the laboratory, the long axis of the FSE does not provide a good proxy for the average orientation of crystals direction of anisotropy because dynamic recrystallization tends to make the LPO rotate faster toward the shear direction [e.g., Zhang and Karato, 1995]. Numerical simulations of LPO evolution in the case of plane deformations led Kaminski and Ribe [2002] to propose that at large strain seismic anisotropy will tend to follow the orientation of the long axis of the FSE that would be reached in the limit of infinite strain, i.e., the ISA.

We calculate the ISA for the two analytical flows using the method described in Kaminski and Ribe [2002], and we compare its orientation with the direction of the fast axis of the hexagonal medium that best fits the elastic tensor (Figure 4). At small strains $\left(E_{0}<1\right)$, the fast axis does not align with the ISA, which is fully consistent with the previous observation that it is aligned with the long axis of the FSE at small strains. At strains $E_{0}>1$, dynamic recrystallization controls the LPO evolution and makes the fast axis rotate toward the ISA rather than to the FSE long axis. Hence, in this second case the ISA is a better proxy than the long axis of the FSE for the hexagonal symmetry axis. As shown by the comparison of Figure 4, the zones of large mismatch between the long axis of the FSE and the fast axis of anisotropy correspond to the zones of good agreement between ISA and fast axis, and vice versa.

\section{Discussion}

Seismic anisotropy is mainly due to crystal deformation in the convective upper mantle. In this framework, its interpretation in terms of mantle flow is not straightforward because the deformation history can be much more complex than the local flow characteristics. Our results provide some guidelines for the interpretation of measured seismic anisotropy.

A first method to interpret seismic anisotropy is the forward calculation of LPO evolution in a numerical flow model proposed for the studied area (e.g., a 3-D dynamic flow in a subduction zone), using a model of plastic deformation, e.g., VPSC if dynamic recrystallization is neglected [Di Leo et al., 2014] or D-Rex if dynamic recrystallization is taken into account [e.g., Faccenda and Capitanio, 2012, 2013]. The anisotropic signature of the calculated LPO can then be compared to seismic data in order to constrain model parameters. However, the required numerical resources may be too large for 3-D flows and/or time-varying flows. In that case, we propose the following approach: (1) to calculate the FSE (hence the natural strain $E_{0}$ ); (2) if $E_{0}<1$, to use the long axis of the FSE as a proxy for the direction of fast axis and the natural stain as a scale for the percentage of anisotropy; and (3) if $E_{0}>1$, to use the direction of the ISA as a proxy for the direction of fast axis and to consider that the percentage of anisotropy has reached a plateau value (depending on the hypothesis on composition and fabric type).

This method takes into consideration the effect of dynamic recrystallization and thus provides a more consistent LPO pattern at high strain by comparison with a method using only the FSE [e.g., Li et al., 2014]. Lev and Hager [2008] introduced a "directors" method which provides a compromise in terms of computational cost between FSE calculation and full mechanical modeling. Their method remains to be tested in non-Newtonian flows and to be compared to the combined FSE/ISA fast method presented here. We postulate that, in general, uncertainties on model parameters in the upper mantle (e.g., exact slip system activities or grain boundary mobility) may be too large for a method more complex than FSE/ISA to be required to interpret seismic anisotropy.

A second method of seismic data interpretation is the inverse approach, in which the direction of fast propagation determined from field measurements is directly taken as the flow direction. This approach is, 

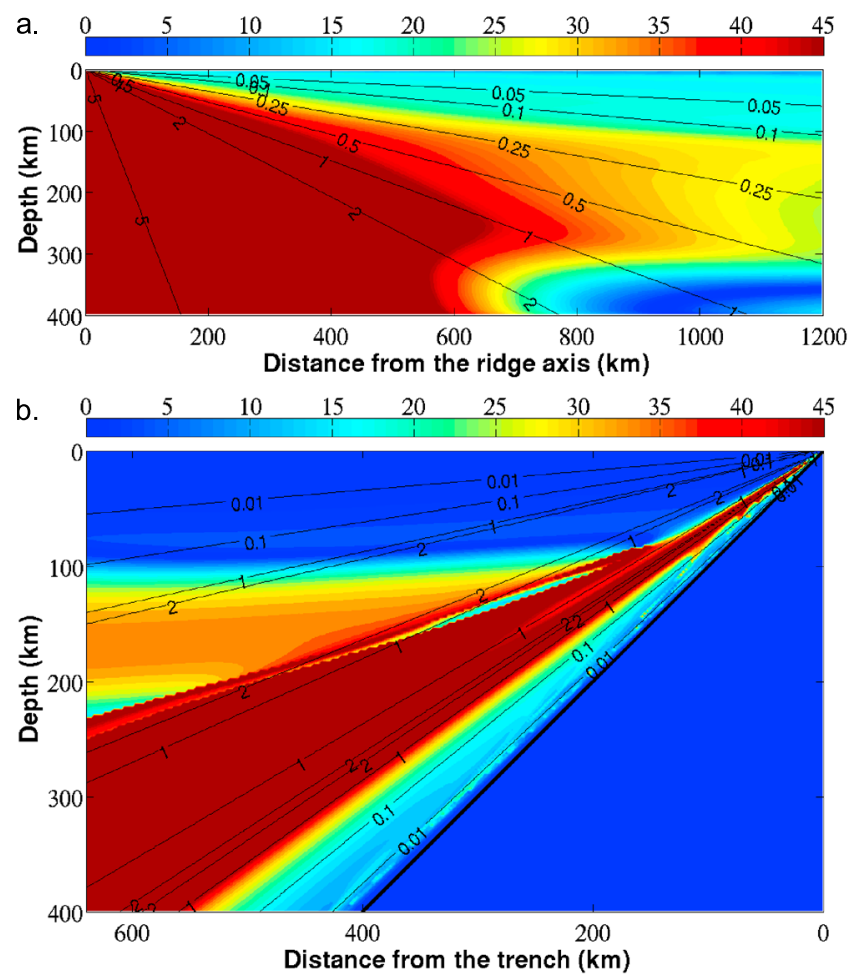

Figure 5. Angle between the hexagonal symmetry axis and the flow direction (color scale, saturated at $45^{\circ}$ ) as a function of the GOL parameter (given as contour lines) in (a) a mid-ocean ridge and (b) a subduction zone.

$$
\mathrm{GOL}=2 \frac{\left|f\left(f^{\prime 2}-f^{\prime \prime}\right)\right|}{\left|f+f^{\prime \prime}\right|\left|f^{2}+f^{\prime 2}\right|},
$$

and we calculate the angle between the fast axis and the flow direction in the two corner flows. The results shown in Figure 5 validate the criterion based on the GOL parameter, but with a more stringent condition than the one proposed by Kaminski and Ribe [2002] for Newtonian flows. In the case of non-Newtonian flows, the direction of the fast axis and the flow direction will be aligned only when the GOL parameter is smaller than 0.1. This is only the case close to the plates, i.e., in the narrow regions where the rate of shear is maximum. We thus suggest that seismic anisotropy is taken as a hint of mantle flow direction only when the zone seismically sampled is close to a plate (such as under the lithosphere or near a subducting slab) and has a narrow extent. SKS splitting parameters, which by definition sample large domains of the upper mantle, should be interpreted in terms of mantle flow with caution in general.

The conclusions reached in this paper are based on the model D-Rex developed by Kaminski et al. [2004], which is still a simplistic model of LPO evolution. Additional parameters are likely to affect the relationship between seismic anisotropy and mantle flow. One kind of parameter is "external," like the local presence of melt or water in the mantle [Holtzman et al., 2003; Jung and Karato, 2001]. A complete forward modeling will be required to interpret seismic anisotropy in such cases as these parameters may change quite abruptly the orientation of the fast axis locally [Kaminski, 2006]. A second kind of parameter is more "internal," e.g., pressure and/or temperature, and are related to fabric transition [Mainprice et al., 2005; Jung et al., 2009] or the relative contribution of dislocation creep and diffusion creep. A recent study has shown for example that diffusion creep may generate a nonrandom LPO in olivine aggregate [Miyazaki et al., 2013], hence should be taken into account in updated models of LPO evolution.

\section{References}

Bai, Q., S. Mackwell, and D. Kohlstedt (1991), High-temperature creep of olivine single crystals. 1. Mechanical results for buffered samples, J. Geophys. Res., 96(B2), 2441-2463.

Batchelor, G. K. (1967), An Introduction to Fluid Dynamics, Cambridge Univ. Press, New York.

Becker, T. W., S. Chevrot, V. Schulte-Pelkum, and D. K. Blackman (2006), Statistical properties of seismic anisotropy predicted by upper mantle geodynamic models, J. Geophys. Res., 111, B08309, doi:10.1029/2005JB004095. 
Blackman, D. K., and J. Kendall (2002), Seismic anisotropy in the upper mantle. 2. Predictions for current plate boundary flow models, Geochem. Geophys. Geosyst., 3(9), 8602, doi:10.1029/2001GC000247.

Browaeys, J. T., and S. Chevrot (2004), Decomposition of the elastic tensor and geophysical applications, Geophys. J. Int., 159(2), 667-678.

Bystricky, M., K. Kunze, L. Burlini, and J.-P. Burg (2000), High shear strain of olivine aggregates: Rheological and seismic consequences, Science, 290(5496), 1564-1567.

Conrad, C. P., and M. D. Behn (2010), Constraints on lithosphere net rotation and asthenospheric viscosity from global mantle flow models and seismic anisotropy, Geochem. Geophys. Geosyst., 11, Q05W05, doi:10.1029/2009GC002970.

Conrad, C. P., M. D. Behn, and P. G. Silver (2007), Global mantle flow and the development of seismic anisotropy: Differences between the oceanic and continental upper mantle, J. Geophys. Res., 112, B07317, doi:10.1029/2006JB004608.

Di Leo, J., A. Walker, Z.-H. Li, J. Wookey, N. Ribe, J.-M. Kendall, and A. Tommasi (2014), Development of texture and seismic anisotropy during the onset of subduction, Geochem. Geophys. Geosyst., 15, 192-212, doi:10.1002/2013GC005032.

Faccenda, M., and F. Capitanio (2012), Development of mantle seismic anisotropy during subduction-induced 3-D flow, Geophys. Res. Lett., 39, L11305, doi:10.1029/2012GL051988.

Faccenda, M., and F. Capitanio (2013), Seismic anisotropy around subduction zones: Insights from three-dimensional modeling of upper mantle deformation and SKS splitting calculations, Geochem. Geophys. Geosyst., 14, 243-262, doi:10.1002/ggge.20055.

Gaboret, C., A. Forte, and J.-P. Montagner (2003), The unique dynamics of the Pacific hemisphere mantle and its signature on seismic anisotropy, Earth Planet. Sci. Lett., 208(3), 219-233.

Hall, C. E., K. M. Fischer, E. Parmentier, and D. K. Blackman (2000), The influence of plate motions on three-dimensional back arc mantle flow and shear wave splitting, J. Geophys. Res., 105(B12), 28,009-28,033.

Holtzman, B., D. Kohlstedt, M. Zimmerman, F. Heidelbach, T. Hiraga, and J. Hustoft (2003), Melt segregation and strain partitioning: Implications for seismic anisotropy and mantle flow, Science, 301(5637), 1227-1230.

Jin, Z., Q. Bai, and D. Kohlstedt (1994), High-temperature creep of olivine crystals from four localities, Phys. Earth Planet. Inter., 82(1), 55-64.

Jung, H., and S.-I. Karato (2001), Water-induced fabric transitions in olivine, Science, 293(5534), 1460-1463.

Jung, H., W. Mo, and H. W. Green (2009), Upper mantle seismic anisotropy resulting from pressure-induced slip transition in olivine, Nat. Geosci., 2(1), 73-77.

Kaminski, E. (2006), Interpretation of seismic anisotropy in terms of mantle flow when melt is present, Geophys. Res. Lett., 33, L02304, doi:10.1029/2005GL024454.

Kaminski, E., and N. Ribe (2001), A kinematic model for recrystallization and texture development in olivine polycrystals, Earth Planet. Sci. Lett., 189(3), 253-267.

Kaminski, E., and N. M. Ribe (2002), Timescales for the evolution of seismic anisotropy in mantle flow, Geochem. Geophys. Geosyst., 3(8), 1-17, doi:10.1029/2001GC000222.

Kaminski, E., N. M. Ribe, and J. T. Browaeys (2004), D-Rex, a program for calculation of seismic anisotropy due to crystal lattice preferred orientation in the convective upper mantle, Geophys. J. Int., 158(2), 744-752.

Karato, S.-I., H. Jung, I. Katayama, and P. Skemer (2008), Geodynamic significance of seismic anisotropy of the upper mantle: New insights from laboratory studies, Annu. Rev. Earth Planet. Sci., 36, 59-95.

Kreemer, C. (2009), Absolute plate motions constrained by shear wave splitting orientations with implications for hot spot motions and mantle flow, J. Geophys. Res., 114, B10405, doi:10.1029/2009JB006416.

Kumazawa, M., and O. L. Anderson (1969), Elastic moduli, pressure derivatives, and temperature derivatives of single-crystal olivine and single-crystal forsterite, J. Geophys. Res, 74(25), 5961-5972.

Lev, E., and B. H. Hager (2008), Prediction of anisotropy from flow models: A comparison of three methods, Geochem. Geophys. Geosyst., 9, Q07014, doi:10.1029/2008GC002032.

Li, Z.-H., J. F. Di Leo, and N. M. Ribe (2014), Subduction-induced mantle flow, finite strain, and seismic anisotropy: Numerical modeling, J. Geophys. Res. Solid Earth, 119, 5052-5076, doi:10.1002/2014JB010996.

Mainprice, D., A. Tommasi, H. Couvy, P. Cordier, and D. J. Frost (2005), Pressure sensitivity of olivine slip systems and seismic anisotropy of Earth's upper mantle, Nature, 433(7027), 731-733.

McKenzie, D. (1979), Finite deformation during fluid flow, Geophys. J. R. Astron. Soc., 58(3), 689-715.

Miyazaki, T., K. Sueyoshi, and T. Hiraga (2013), Olivine crystals align during diffusion creep of Earth's upper mantle, Nature, 502(7471), 321-326.

Montagner, J.-P. (1994), Can seismology tell us anything about convection in the mantle?, Rev. Geophys., 32(2), 115-137.

Montagner, J.P., and H.-C. Nataf (1988), Vectorial tomography. I. Theory, Geophys. J., 94(2), 295-307.

Nicolas, A., and N. I. Christensen (1987), Formation of anisotropy in upper mantle peridotites-A review, in Composition, Structure and Dynamics of the Lithosphere-Asthenosphere System, Geodyn. Ser., vol. 16, edited by K. Fuchs and C. Froidevaux, pp. 111-123, AGU, Washington, D. C.

Nicolas, A., F. Boudier, and A. Boullier (1973), Mechanisms of flow in naturally and experimentally deformed peridotites, Am. J. Sci, 273(10), 853-876.

Poirier, J.-P. (1985), Creep of Crystals, 260 pp., Cambridge Univ. Press, Cambridge, New York.

Ribe, N. M. (1989), Seismic anisotropy and mantle flow, J. Geophys. Res., 94(B4), 4213-4223.

Ribe, N. M. (1992), On the relation between seismic anisotropy and finite strain, J. Geophys. Res., 97(B6), 8737-8747.

Savage, M. (1999), Seismic anisotropy and mantle deformation: What have we learned from shear wave splitting?, Rev. Geophys., 37(1), 65-106.

Tanimoto, T., and D. L. Anderson (1984), Mapping convection in the mantle, Geophys. Res. Lett., 11(4), 287-290.

Tommasi, A. (1998), Forward modeling of the development of seismic anisotropy in the upper mantle, Earth Planet. Sci. Lett., 160(1), 1-13.

Tovish, A., G. Schubert, and B. P. Luyendyk (1978), Mantle flow pressure and the angle of subduction: Non-Newtonian corner flows, J. Geophys. Res., 83(B12), 5892-5898.

Zhang, S., and S.-I. Karato (1995), Lattice preferred orientation of olivine aggregates deformed in simple shear, Nature, 375(6534), 774-777. 